\title{
Evaluation of eicosanoids in nasal lavage as biomarkers of inflammation in patients with allergic rhinitis
}

\author{
Maciej Ciebiada ${ }^{1}$, Paweł Górski ${ }^{2}$, Adam Antczak $^{1}$
}

${ }^{1}$ Department of General and Oncological Pulmonology, Medical University of Lodz, Lodz, Poland

${ }^{2}$ Department of Pneumonology and Allergy, Medical University of Lodz, Lodz, Poland

Submitted: 10 February 2013

Accepted: 30 May 2013

Arch Med Sci 2014; 10, 6: 1123-1128

DOI: 10.5114 /aoms.2015.47655

Copyright $\odot 2014$ Termedia \& Banach

\section{Abstract}

Introduction: Cysteinyl leukotrienes (cys-LTs), 8-isoprostane and prostaglandin E2 ( $\mathrm{PGE}_{2}$ ) constitute fundamental mediators in allergic inflammation; therefore we wanted to determine the utility of $\mathrm{PGE}_{2}, 8$-isoprostane and cys-LT levels in nasal lavage as biomarkers of allergic inflammation.

Material and methods: Twenty-one patients with allergic rhinitis (AR) were included on the basis of a positive history of AR symptoms and positive results of skin prick tests to grass pollen allergens. The main exclusion criteria were: uncontrolled asthma, nasal polyps, respiratory infection, tuberculosis, neoplastic and autoimmune diseases, current smoking and immunotherapy. Both outside the pollen season and at the height of the pollen season, total nasal symptom score (TNS-4) was evaluated and the levels of cys-LTs, 8-isoprostane and $\mathrm{PGE}_{2}$ were measured in nasal lavage fluid (NALF).

Results: Natural allergen stimulation resulted in a significant increase of TNS-4 $(p<0.001)$ and nasal eosinophilia $(p<0.001)$. The concentration of $\mathrm{PGE}_{2}$ dominated in the NALF outside the pollen season and decreased significantly at the height of natural exposure $(p<0.01)$. In contrast, lower baseline concentrations of cys-LTs and 8-isoprostane increased significantly upon allergen stimulation $(p<0.05)$. There was a significant correlation between mean concentration of $\mathrm{PGE}_{2}$ and eosinophil number in NALF $(r=0.67$, $p=0.0439$ ).

Conclusions: The NALF concentrations of cys-LTs and 8-isoprostane change simultaneously with TNS-4 and nasal eosinophilia. However, due to the lack of any significant correlation, their utility as markers of allergic rhinitis should be warily considered. The decrease of $\mathrm{PGE}_{2}$ concentration in NALF which correlated with nasal eosinophilia may participate in escalation of allergic inflammation and needs further evaluation.

Key words: nasal lavage, allergic rhinitis, leukotrienes, prostaglandins, eicosanoids, biomarkers.

\section{Introduction}

There have been many attempts to find non-invasive and simple to execute methods for evaluation of respiratory diseases. Determining the concentration or activity of biological markers in materials obtained from patients or people with risk factors was used in the diagnosis of sarcoidosis, interstitial lung diseases, lung cancer or chronic obstructive pulmonary disease (COPD) [1-4]. Finding specific and sensitive markers of the disease would allow rapid diagnosis, assessment of the disease stage,

\author{
Corresponding author: \\ Maciej Ciebiada MD, PhD \\ Department \\ of Pneumonology and Allergy \\ Medical University of Lodz \\ 22 Kopcińskiego St \\ 90-153 Lodz, Poland \\ Phone: +48 426787505 \\ Fax: +48426782129 \\ E-mail: maciej_ciebiada@ \\ op.pl
}


and estimation of the response to treatment. Unfortunately, in allergic rhinitis such specific markers of airways inflammation have not yet been clearly defined.

However oxidative stress is increased [5] and cyclooxygenase-2 (COX) and 5-lipoxygenase (5LOX) are overexpressed in the inflammatory milieu $[6,7]$. Therefore we hypothesized that 8-isoprostane, prostaglandin $\mathrm{E}_{2}\left(\mathrm{PGE}_{2}\right)$ and cysteinyl leukotrienes (cys-LTs), which constitute the end products of nonenzymatic and enzymatic metabolism of arachidonic acid, would be detectable in nasal lavage collected from patients with allergic rhinitis and could serve as markers of allergic in flammation in the nasal mucosa.

In this study we assessed the levels of $\mathrm{PGE}_{2}$, cys-LTs and 8-isoprostane in the nasal lavage of patients with seasonal allergic rhinitis at baseline, outside the pollen season and during natural allergen stimulation, at the height of the pollen season. In addition we wanted to assess the relationship between the levels of PGE ${ }_{2}$, cys-LTs, 8-isoprostane and the symptom score as well as nasal eosinophilia in patients with seasonal allergic rhinitis.

\section{Material and methods}

In this single center, open study, we enrolled 21 patients with seasonal allergic rhinitis (AR). Allergic rhinitis was diagnosed in accordance with the allergic rhinitis and its impact on asthma guidelines [8]. All patients had to have at least a two-year history of pollinosis due to the sensitization to grass pollen allergens with the positive skin prick test (SPT) result and high concentration of IgE specific to grass pollen. Well-controlled asthma only with short acting inhaled beta agonists did not constitute an exclusion criterion. The main exclusion criteria comprised: asthma treated with inhaled or systemic glucocorticosteroids, topical or systemic treatment with glucocorticosteroids due to other disease, nasal polyps, respiratory tract infection for at least 4 weeks preceding the study, breast feeding, pregnancy, tuberculosis, neoplastic and autoimmunologic diseases, current smoking and specific allergen immunotherapy.

Table I. Study population

\begin{tabular}{|lc|}
\hline Parameter & Results \\
\hline Number of patients & 21 \\
\hline Age [years] & $34.71 \pm 3.2$ \\
\hline Sex $(\mathrm{M}: \mathrm{F})$ & $9: 12$ \\
\hline FEV & (\% of predicted value) \\
\hline Duration of allergic rhinitis [years] & $89.9 \pm 7.6$ \\
\hline Values given as mean $\pm S D$. & $13.4 \pm 6.8$ \\
\hline
\end{tabular}

After obtaining written consent from each participant, the patients were asked to withhold any anti-allergic therapy for 2 weeks prior to the study (a 2-week run-in) and were supplied with the diaries. The patients were to record the symptoms every day, with particular consideration of the symptoms of allergic rhinitis. Then, at the first visit still outside the pollen season, the diaries were evaluated, physical examination and spirometry were performed, and nasal lavage was collected. Next the patients were asked to fill in their diaries during the pollen season when the predicted concentration of pollen in the air was the highest: 2 weeks before, at the height of the pollen season, and 2 weeks after the peak of the pollen season. The course of the pollen season (start, the height, and termination) was predicted on the basis of retrospective analysis of the grass pollen concentration in the air during the last 5 pollen seasons.

At the height of the pollen season, the patients underwent physical examination, and nasal lavage (NAL) was collected. The characteristics of the study population are presented in Table I.

The study protocol was approved by the local Ethics Committee.

\section{Collection of nasal lavage}

Collection of nasal lavage was performed as previously described [9]. Briefly, a syringe with gum rubber serving as a seal was connected to one nostril, and $5 \mathrm{ml}$ of sterile, isotonic $\mathrm{NaCl}$ at room temperature was instilled into the nasal cavity. The patients were asked to sit motionless with the head bent to the chest for $5 \mathrm{~min}$. Then, fluid was aspirated to the syringe by gentle suction. The procedure was repeated in the opposite nostril.

Lavage was centrifuged (10 min, 300x, room temperature) and the supernatant was collected for further analysis. The cells were counted with the light microscope and the numbers of eosinophils were presented as a percentage of total cell count.

\section{Leukotrienes}

The lavage concentration of cysteinyl leukotrienes (cys-LTs) was examined using an enzyme immunoassay (EIA) kit (Cayman Chemical, MI, USA) as previously described [2]. The minimum detectable concentration was $13 \mathrm{pg} / \mathrm{ml}$ [10].

\section{8-Isoprostane}

8-Isoprostane concentration in nasal lavage was determined with the EIA kit (Cayman Chemical, MI, USA) as previously presented [3] with an antiserum which had $100 \%$ cross-reactivity with 8 -isoprostane, $0.77 \%$ with prostaglandin $\mathrm{F} 1,0.66 \%$ with prostaglandin $\mathrm{F} 3$, and $0.31 \%$ with prostaglandin E1. The detection limit of the assay was $5 \mathrm{pg} / \mathrm{ml}$. 


\section{Prostaglandin $\mathrm{E}_{2}$}

To measure the concentration of prostaglandin $E_{2}$ in nasal lavage the EIA kit (Cayman Chemical, MI, USA) was used with an antiserum which had $100 \%$ cross-reactivity with $\mathrm{PGE}_{2}, 43 \%$ with $\mathrm{PGE}_{3}, 18.7 \%$ with $\mathrm{PGE}_{1}$, and $0.1 \%$ each with $\mathrm{PGF}_{2 \alpha}$, $P G A_{1}$, and $P G A_{2}$. The minimum detection limit was $1.5 \mathrm{pg} / \mathrm{ml}$.

\section{Lung function test}

Pulmonary functions were measured in all participants at the screening visit, outside the pollen season, with a computer-assisted spirometer (Lung Test 1000, Mes Dymek, Poland) according to American Thoracic Society guidelines [11]. The best value of three maneuvers was noted and expressed as a percentage of the predicted value. The methacholine challenge test was performed as previously described [12], and the concentration of methacholine which produced a $20 \%$ fall of forced expiratory volume in $1 \mathrm{~s}\left(\mathrm{FEV}_{1}\right)$ was calculated (PC20). Patients with PC20 lower than $8 \mathrm{mg}$ were considered to have bronchial hyperresponsiveness.

\section{Symptom score}

Both outside the pollen season and during the symptomatic period evoked by natural allergen stimulation, the patients were asked to write down AR symptoms every day. The symptoms included nasal itching, sneezing, nasal blockage and rhinorrhea, each graded 0-3 (0 no symptoms, 1 mild symptoms with minimal inconvenience, 2 moderate symptoms, and 3 severe symptoms that interfere with everyday activity and/or sleep). Then, the combined result which sums the scores of each symptom was calculated and presented as the total nasal symptom score (TNS-4). The maximum TNS-4 was 12 .

\section{Skin prick tests and specific IgE}

Skin prick tests were performed with a commercially available set of allergens (Allergopharma J. Ganzer KG, Reinbeck, Germany), with histamine
(10 $\mathrm{mg} / \mathrm{ml})$ as a positive control and diluent as a negative one, while the serum concentration of IgE to grass pollen was determined using the Pharmacia UniCap System according to the manufacturer's protocol (Pharmacia \& Upjohn, Diagnostics $A B$ ). IgE levels had to be higher than $1.5 \mathrm{kU} / \mathrm{l}$. The sensitivity for detection was $0.15 \mathrm{kU} / \mathrm{l}$.

\section{Statistical analysis}

For normally distributed data, values were expressed as the mean \pm standard error of the mean, whereas for data which were not normally distributed, the values were presented as the median with $25^{\text {th }}$ and $75^{\text {th }}$ percentiles. A Wilcoxon test was used to compare paired results that were not normally distributed, and correlations between variables were evaluated by Spearman's test. With a sample size of 21 and $\alpha=0.05$ as well as a sigma value adequate to each parameter assessed in the study, the power of the test reached $100 \%$. Values of $p<0.05$ was considered significant (GraphPad Prism 5, San Diego, CA).

\section{Results}

The levels of cys-LTs were below the detection limit in 4 patients, whereas the concentration of 8-isoprostane was below the detection limits in 1 patient outside the pollen season and in 5 patients at the height of the pollen season. Prostaglandin $E_{2}$ was not detected in 3 unexposed patients.

Outside the pollen season the concentration of $\mathrm{PGE}_{2}$ was the highest in nasal aspirate and the concentration of 8-isoprostane was the lowest when compared with other mediators (Table II, Figure 1).

Concentrations of cys-LTs and 8-isoprostane in nasal lavage increased significantly at the height of the pollen season ( $p<0.05$ for both cys-LTs and 8-isoprostane), whereas the levels of $\mathrm{PGE}_{2}$ significantly decreased during the pollen exposure $(p<0.01)$ (Table II, Figure 1), and then it was detectable only in 10 patients.

The cellular profile of nasal lavage was as follows: neutrophils $21.6 \pm 4.67 \%$, macrophages $0 \%$,

Table II. Concentrations of cys-LTs, PGE 2 and 8-isoprostane in nasal lavage of patients with allergic rhinitis both outside the pollen season (A) and at the height of the pollen season (B) (in pg/ml). For normally distributed data, values are presented as mean \pm SEM. For non-normally distributed data, values are additionally presented as median with $25^{\text {th }}$ and $75^{\text {th }}$ percentiles

\begin{tabular}{|c|c|c|c|c|c|c|c|c|}
\hline & \multicolumn{2}{|c|}{ cys-LTs } & \multicolumn{2}{|c|}{$\mathrm{PGE}_{2}$} & \multicolumn{2}{|c|}{ 8-Isoprostane } & \multicolumn{2}{|c|}{ Eosinophils (\%) } \\
\hline & A & B & A & B & A & B & A & B \\
\hline AR patients & $\begin{array}{c}98.14 \pm 13.4 \\
78 \\
(36.5-153.5)\end{array}$ & $\begin{array}{l}153.1 \\
\pm 20.1\end{array}$ & $\begin{array}{l}159.9 \\
\pm 27.9\end{array}$ & $\begin{array}{c}91.95 \pm 25.15 \\
61.5 \\
(0-200)\end{array}$ & $\begin{array}{c}32.14 \\
\pm 3.6\end{array}$ & $\begin{array}{c}43.39 \\
\pm 5.0\end{array}$ & $\begin{array}{c}6.91 \\
\pm 0.79\end{array}$ & $\begin{array}{c}10.76 \\
\pm 1.28^{*}\end{array}$ \\
\hline
\end{tabular}




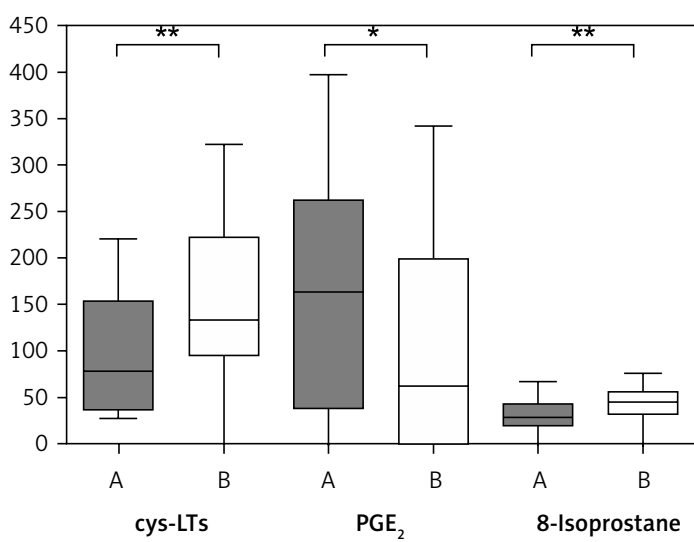

Figure 1. Levels of cys-LTs, PGE 2 and 8-isoprostane in nasal lavage of patients with allergic rhinitis outside the pollen season (A) and at the height of the pollen season (B)

${ }^{*} p<0.01,{ }^{* *} p<0.05$. Bars represent the values from the $25^{\text {th }}$ to $75^{\text {th }}$ percentile, the middle line represents the median, and whiskers extend from the minimum to the maximum value.

Table III. Correlation between concentrations of cys-LTs, 8-isoprostane, PGE $_{2}$ and nasal eosinophilia in nasal lavage measured at the height of the pollen season

\begin{tabular}{|lcc|}
\hline Variable & \multicolumn{2}{c|}{ Correlations } \\
\cline { 2 - 3 } & $r$ & $p$ \\
\hline cys-LTs & 0.106 & 0.68 \\
\hline 8-Isoprostane & 0.13 & 0.61 \\
\hline PGE $_{2}$ & 0.67 & 0.039 \\
\hline
\end{tabular}

lymphocytes $6.6 \pm 1.4 \%$, monocytes $0.05 \pm 0.05 \%$, eosinophils $6.9 \pm 0.79 \%$, basophils 0 , epithelium $65.5 \pm 5.7 \%$. Natural stimulation with grass pollen resulted in a prompt increase in the number of nasal eosinophils $(p<0.001)$ (Table II).

There was a strong positive correlation between $\mathrm{PGE}_{2}$ in nasal lavage and the number of eosinophils at the height of the pollen season $(r=0.67, p=0.039)$. We did not find any correlation either for cys-LTs or for 8-isoprostane $(r=0.106$, $p=0.68 ; r=0.13, p=0.61$ for cys-LTs and 8-isoprostane respectively) (Table III).

We found no correlation between the values of $\mathrm{FEV}_{1}$ and concentrations of eicosanoids.

Finally, natural pollen exposure resulted in a significant increase of the mean TNS-4 score (3.04 $\pm 2.23,5.65 \pm 2.97, p<0.001$ for baseline and the height of the pollen season respectively); however, we did not find any correlation between TNS-4 and eicosanoid concentrations.

\section{Discussion}

The nasal mucosa is the first protective barrier of the body providing a platform for the first line immunologic responses. Being metabolically active, mucosal epithelium has been shown to regulate repair and contribute to inflammatory processes. In this context, the epithelium releases a package of growth factors, adhesion molecules and pro-inflammatory cytokines [13] as well as potent anti-inflammatory mediators such as ni-

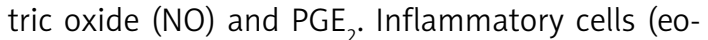
sinophils, lymphocytes, mast cells) that reside in or infiltrate the mucous membrane intensify the allergic response and through rapidly released (histamine) or synthesized mediators (cytokines, metabolites of arachidonic acid) produce severe symptoms of rhinitis that may significantly deteriorate patients' quality of life [14]. Since allergic rhinitis is the most common allergy in urbanized countries, there is a great need for new, specific and validated biomarkers that are predictive of allergic inflammation severity in the airway epithelium and could help to determine treatment.

Although the measurement of NO in the air exhaled orally may serve as a useful marker in airway inflammation monitoring in asthma $[15,16]$, up till now there are no standardized and reliable methods for NO measurement in the nose [17]. Furthermore, function tests that measure nasal patency or volumes may identify nasal congestion, which is one, selected symptom of allergic rhinitis, which poorly reflects the intensity of inflammation [18]. In this context, metabolites of arachidonic acid seemed to be potential markers of inflammation in allergic rhinitis.

It has been demonstrated that cys-LTs, 8-isoprostane and $P G E_{2}$ found in the airways of $A R$ patients $[19,20]$ contribute to the progression and modulation of the allergic response. Cys-LTs are potent proinflammatory lipid mediators which increase nasal blood flow and escalate leukocyte accumulation, their activation and survival at the site of inflammation [21]. 8-Isoprostane originates as a dominant secondary product of lipid peroxidation, and its measurement reflects quantitatively the activity of oxidative stress in the inflammatory milieu $[22,23]$. Contrariwise, $P G E_{2}$, which is both a pro- and an anti-inflammatory mediator in allergic inflammation, is believed to exert a suppressive function [24]. Prostaglandin $E_{2}$ not only inhibits leukocyte chemotaxis and release of mediators but also enhances epithelial cell survival [21], regulates production of proinflammatory cytokines [25] and up-regulates some anti-inflammatory cytokines, including IL-10 [26]. Prostaglan$\operatorname{din} \mathrm{E}_{2}$ regulates angiogenesis [27] and relaxes airway smooth muscle [28]. When inhaled, it protects against bronchoconstriction [29].

Cys-LTs and 8-isoprostane concentrations were found to be elevated in exhaled breath condensate (EBC) and bronchoalveolar lavage fluid (BALF) 
in patients with asthma, lung cancer [30], and sarcoidosis [4], as well as in EBC [31] and nasal lavage [31, 32] in patients with seasonal allergic rhinitis. Prostaglandin $\mathrm{E}_{2}$ was detected in $\mathrm{EBC}$ and BALF of patients with sarcoidosis [30], chronic obstructive pulmonary disease [19] and lung cancer $[5,6,27]$, and in nasal lavage in patients with allergic rhinitis [20].

Our study demonstrates that cys-LTs, 8-isoprostane and $\mathrm{PGE}_{2}$ are detectable in nasal lavage and their concentrations significantly vary during natural allergen stimulation. While baseline status is associated with low NAL concentrations of 8-isoprostane and cys-LTs and high concentration of $\mathrm{PGE}_{2}$, natural allergen stimulation reverses this state, increasing cys-LTs and 8-isoprostane simultaneously reducing $\mathrm{PGE}_{2}$.

Inflammatory cells within the nasal mucosa of AR patients are in an activated state and have the capability of generating leukotriene mediators that are also of potential relevance to symptom generation. The immediate nasal response raises cys-LTs and 8-isoprostane levels in nasal lavage fluids [33], which is accompanied by deterioration of nasal symptoms. The patient's recovery is then paralleled by a decline of NAL concentrations of lipid mediators [34]. Our study also showed that the intensification of symptoms was accompanied by an increase of cys-LTs and 8-isoprostane levels in NAL, whereas the asymptomatic period was associated with a significant decrease of both mediators. A noteworthy fact is that allergen stimulation resulted in a significant decrease of $\mathrm{PGE}_{2}$ levels, whereas a lack of stimulation restored $\mathrm{PGE}_{2}$ concentration. Our study has demonstrated this for the first time. It is in contrast with the results of Sugimoto's study [20], where nasal provocation tests resulted in a significant increase of NAL PGE. Furthermore, simulation with nonspecific irritants (cold, dry air) resulted in a significant increase of $\mathrm{PGE}_{2}$ in healthy subjects [35]. However, Koren did not find such an increase in patients stimulated with ozone [36]. The results of these studies may support a dual role of $\mathrm{PGE}_{2}$ in inflammation. Since natural stimulation with an allergen resulted in a decrease of $\mathrm{PGE}_{2}$ at the same time as severe symptoms and nasal eosinophilia, it is plausible that the impairment of biosynthesis of this prostaglandin could be an additional cause of exacerbation.

In conclusion, as we have demonstrated, natural allergen stimulation increases both cys-LT and 8-isoprostane concentrations in NAL as well as nasal eosinophilia and the symptom score in AR patients. The decrease in concentration of $P G E_{2}$, which possesses anti-inflammatory properties, at the height of the pollen season may constitute another background for an outbreak of inflammation and strengthen AR symptoms. As we did not find any significant positive correlation between the levels of 8-isoprostane, cys-LTs in NAL and values of the TNS-4 score and nasal eosinophilia, the usefulness of measurements of these lipid mediators as indices of inflammation severity should be considered warily. Furthermore, targeting the $\mathrm{PGE}_{2}$-dependent signals in the airways should be better evaluated, since it may constitute another new therapeutic strategy.

\section{Acknowledgments}

This study was supported by the Medical University of Lodz.

\section{References}

1. Ciebiada M, Górski P, Antczak A. Eicosanoids in exhaled breath condensate and bronchoalveolar lavage fluid of patients with primary lung cancer. Dis Markers 2012; 32: 329-35.

2. Antczak A, Montuschi P, Kharitonov SA, Gorski P, Barnes PJ. Increased exhaled cysteinyl-leukotrienes and 8-isoprostane in aspirin-induced asthma. Am J Respir Crit Care Med 2002; 166: 301-6.

3. Montuschi P, Corradi M, Ciabattoni G, Nightingale J, Kharitonov SA, Barnes PJ. Increased 8-isoprostane, a marker of oxidative stress, in exhaled condensate of asthma patients. Am J Respir Crit Care Med 1999; 160: 216-20.

4. Piotrowski WJ, Antczak A, Marczak J, Nawrocka A, Kurmanowska Z, Górski P. Eicosanoids in exhaled breath condensate and BAL fluid of patients with sarcoidosis. Chest 2007; 132: 589-96.

5. Katsabeki-Katsafli A, Kerenidi T, Kostikas K, et al. Serum vascular endothelial growth factor is related to systemic oxidative stress in patients with lung cancer. Lung Cancer 2008; 60: 271-6.

6. Carpagnano GE, Spanevello A, Palladino GP, et al. Cigarette smoke and increased COX-2 and survivin levels in exhaled breath condensate of lung cancer patients: how hot is the link? Lung Cancer 2010; 67: 108-13.

7. Luo M, Lee S, Brock TG. Leukotriene synthesis by epithelial cells. Histol Histopathol 2003; 18: 587-95.

8. Bousquet J, Khaltaev N, Cruz AA, et al. Allergic Rhinitis and its Impact on Asthma (ARIA) 2008 update (in collaboration with the World Health Organization, GA(2)LEN and AllerGen). Allergy 2008; 63: 8-160.

9. Schäper C, Gustavus B, Koch B, et al. Effects of fexofenadine on inflammatory mediators in nasal lavage fluid in intermittent allergic rhinitis. J Investig Allergol Clin Immunol 2009; 19: 459-64.

10. Montuschi P, Ragazzoni E, Valente E, et al. Validation of leukotriene B4 measurements in exhaled breath condensate. Inflamm Res 2003; 52: 69-73.

11. Pellegrino R, Viegi G, Brusasco V, et al. Interpretative strategies for lung function tests. Eur Respir J 2005; 26 : 948-68.

12. Cockroft DW, Killian DN, Mellon JJ, Hargreave FE. Bronchial reactivity to inhaled histamine: a method and clinical survey. Clin Allergy 1977; 7: 235-43.

13. Gorska-Ciebiada M, Ciebiada M, Gorska MM, Gorski P, Grzelewska-Rzymowska I. Intercellular adhesion molecule 1 and tumor necrosis factor alpha in asthma and persistent allergic rhinitis: relationship with disease severity. Ann Allergy Asthma Immunol 2006; 97: 66-72. 
14. Ciebiada M, Ciebiada MG, Kmiecik T, DuBuske LM, Gorski P. Quality of life in patients with persistent allergic rhinitis treated with montelukast alone or in combination with levocetirizine or desloratadine. J Investig Allergol Clin Immunol 2008; 18: 343-9.

15. Pedrosa M, Cancelliere N, Barranco P, López-Carrasco V, Quirce S. Usefulness of exhaled nitric oxide for diagnosing asthma. J Asthma 2010; 47: 817-21.

16. Lis G, Twarduś K. Allergic and nonallergic rhinitis in schoolchildren. Part II. Relationship to spirometric parameters, bronchial hyperresponsiveness and biomarkers of allergic inflammation. Postep Derm Alergol 2011; 6: 455-61.

17. Moody A, Fergusson W, Wells A, Bartley J, Kolbe J. Nasa levels of nitric oxide as an outcome variable in allergic upper respiratory tract disease: Influence of atopy and hayfever on nNO. Am J Rhinol 2006; 20: 425-9.

18. Keck T, Wiesmiller K, Lindemann J, Rozsasi A. Acoustic rhinometry in nasal provocation test in perennial allergic rhinitis. Eur Arch Otorhinolaryngol 2006; 263: 910-6.

19. Corhay JL, Henket M, Nguyen D, Duysinx B, Sele J, Louis R. Leukotriene B4 contributes to exhaled breath condensate and sputum neutrophil chemotaxis in COPD. Chest 2009; 136: 1047-54.

20. Sugimoto M, Sugiyama S, Yanagita N, Ozawa T. Laser high performance liquid chromatography determination of prostaglandins in nasal lavage fluid in allergic rhinitis. Clin Exp Allergy 1994; 24: 324-9.

21. Huang SK, Peters-Golden M. Eicosanoid lipid mediators in fibrotic lung diseases: ready for prime time? Chest 2008; 133: 1442-50.

22. Dalaveris E, Kerenidi T, Katsabeki-Katsafli A, et al. VEGF, TNF-alpha and 8-isoprostane levels in exhaled breath condensate and serum of patients with lung cancer. Lung Cancer 2009; 64: 219-25.

23. Cruz MJ, Sánchez-Vidaurre S, Romero PV, Morell F, Munoz X. Impact of age on $\mathrm{pH}$, 8-isoprostane, and nitrogen oxides in exhaled breath condensate. Chest 2009; 139: 462-67.

24. Shinomiya S, Naraba H, Ueno A, et al. Regulation of TNFalpha and interleukin-10 production by prostaglandins $\mathrm{I}(2)$ and $\mathrm{E}(2)$ : studies with prostaglandin receptor-deficient mice and prostaglandin E-receptor subtype-selective synthetic agonists. Biochem Pharmacol 2001; 61: 1153-60.

25. leFever A, Funahashi A. Elevated prostaglandin E2 levels in broncholalveolar lavage fluid of patients with bronchogenic carcinoma. Chest 1990; 98: 1397-402.

26. Rola-Pleszczynski M, Stankova J. Cytokine gene regulation by PGE2, LTB4 and PAF. Mediators Inflamm 1992; 1: 5 -8.

27. Amano $\mathrm{H}$, Hayashi I, Endo $\mathrm{H}$, et al. Host prostaglandin E(2)-EP3 signaling regulates tumor-associated angiogenesis and tumor growth. J Exp Med 2003; 197: 221 32.

28. Knight DA, Stewart GA, Thompson PJ. PGE2 but not prostacyclin inhibits histamine-induced contraction of human bronchial smooth muscle. Eur J Pharmacol 1995; 272: 13-9.

29. Gauvreau GM, Watson RM, O’Byrne PM. Protective effects of inhaled PGE2 on allergen-induced airway responses and airway inflammation. Am J Respir Crit Care Med 1999; 159: 31-6.

30. Antczak A, Piotrowski WJ, Marczak J, Ciebiada M, Gorski P, Barnes PJ. Correlation between eicosanoids in bronchoalveolar lavage fluid and in exhaled breath condensate. Dis Markers 2011; 30: 213-20.
31. Gratziou C, Rovina N, Makris M, Simoes DC, Papapet ropoulos A, Roussos C. Breath markers of oxidative stress and airway inflammation in Seasonal Allergic Rhinitis. Int J Immunopathol Pharmacol 2008; 21: 949-57.

32. Tanou K, Koutsokera A, Kiropoulos TS, et al. Inflammatory and oxidative stress biomarkers in allergic rhinitis: the effect of smoking. Clin Exp Allergy 2009; 39: 345-53.

33. Pipkorn U, Proud D, Lichtenstein LM, et al. Effect of short term systemic glucocorticoid treatment on human nasal mediator release after antigen challenge. J Clin Invest 1987; 80: 957-61.

34. Miadonna A, Tedeschi A, Leggieri E, et al. Behaviour and clinical relevance of histamine and leukotrienes C4 and B4 in grass-polleninduced rhinitis. Am Rev Respir Dis 1987; 136: 357-62.

35. Mohammadian P, Schaefer D, Hummel T, Kobal G. Experimentally induced nasal irritation. Rhinology 1999 37: $175-8$.

36. Koren HS, Hatch GE, Graham DE. Nasal lavage as a tool in assessing acute inflammation in response to inhaled pollutants. Toxicology 1990; 60: 15-25. 